\title{
APPLYING TEXTILE MATERIALS FOR THE DESIGN OF ANTENNAS FOR WIRELESS BODY AREA NETWORKS.
}

\author{
Anneleen Tronquo ${ }^{1}$, Hendrik Rogier $^{1}$, Carla Hertleer $^{2}$, and Lieva Van Langenhove ${ }^{2}$ \\ ${ }^{1}$ Ghent University, Department of Information Technology, Sint-Pietersnieuwstraat 41, 9000 GENT, Belgium, \\ Tel: 32926433 43, fax: 32926499 69, e-mail: hendrikr@intec.UGent.be. \\ ${ }^{2}$ Ghent University, Department of Textiles, Technologiepark 907, 9052 ZWIJNAARDE, Belgium
}

\begin{abstract}
The design and manufacturing of several new textilebased rectangular-ring antennas for body area networks operating in the ISM-band $(2.4 \mathrm{GHz}-2.4835 \mathrm{GHz})$ is described. Fleece fabric is applied as antenna substrate, whereas the conducting antenna plane and ground plane are constructed based on a commercially available electrotextile called Flectron. We compare the characteristics of two prototypes that were assembled using different glueing techniques. The influence of bending on the characteristics is investigated. The proposed antennas are the first circularly polarised antennas that cover a bandwidth of more than $190 \mathrm{MHz}$, using a substrate material with a thickness less than $3 \mathrm{~mm}$. The antennas provide sufficient antenna gain for use in practical wireless links. They allow direct integration into garments.
\end{abstract}

Key words: microstrip patch antenna; elektrotextiles; Wearable Textile Systems.

\section{INTRODUCTION}

The trend of integrating more and more functionality into garments has led to a new range of applications known as 'Wearable Textile Systems' [1,2]. They consist of systems integrated into clothes offering functions such as monitoring of life signs [3], positioning and activity monitoring, and detection of parameters of the environment [4], e.g. temperature or toxic gases. The need for sending the measured parameters to a control centre over a wireless link, resulted in the concept of wearable antennas $[5,6,7,8]$. Those inexpensive antennas do not disturb the movements of the person wearing them, as they are flexible and light weight.

The introduction of new wireless protocols for body area networks and personal area networks (BANs and PANs) operating in the $2.45-\mathrm{GHz}$ band, based on the Zigbee (IEEE 802.15.4), Bluetooth (IEEE 802.15), WiFi (IEEE $802.11 \mathrm{~b} / \mathrm{g}$ ), Wireless USB, and WiMAX (IEEE 802.16a) protocols, poses some real challenges to antenna design based on textile materials. As the bandwidth of the Industrial Scientific Medical (ISM)-band is quite large, special techniques are needed in order to cover the complete communication band. In [9], we have presented a rectangular-ring textile antenna, where Flectron is used to construct the conductive antenna patch and ground plane. In [10], we have compared different electrotextiles for the construction of rectangular-ring textile antennes. The rectangular-ring antenna provides a large bandwidth by placing the feeding point close to the diagonal of the patch. This allows the excitation of two modes with slightly different resonance frequencies, thereby increasing the bandwidth compared to standard microstrip patch antennas. In [9], glueing and stitching was applied to assemble the different textile layers, being antenna ground plane, the non-conductive substrate and the antenna plane. This type of construction process is quite labour-intensive, expensive and difficult to control and reproduce.

In this article we introduce a new assembly technique based on a adhesive sheet and ironing, without the need of additional stitching. The effect of the adhesive sheet on the different antenna parameters is investigated. The performance of the textile antenna prototypes based on the new construction technique is compared to the antenna prototype described in [9].

\section{TEXTILE ANTENNA DESIGN}

\subsection{Material selection and characterisation}

The first step in the design of a textile antenna consists of choosing appropriate materials for the antenna substrate and the conducting parts. As antenna substrate, we rely on a Fleece fabric with a thickness of $2.56 \mathrm{~mm}$. Because of the inherent piled structure, the Fleece contains much air and therefore the permittivity is close to one. Low permittivity of the antenna substrate allows the design of antennas with a large gain and a high efficiency. For the conducting parts we choose Flectron, a thin copper plated high quality ripstop fabric, because of its low surface resistivity (less then $0.1 \Omega / s q$ ) and its large tem- 
perature range $\left(-40^{\circ} \mathrm{C}\right.$ to $\left.180^{\circ} \mathrm{C}\right)$, which enables us to solder the connector without burning holes in the electrotextile. The relative permittivity of the Fleece fabric has been derived by comparing the measured and simulated resonance frequencies of a rectangular patch antenna and has been found to be 1.25 . The losses in the Fleece fabric are characterised by measuring the link budget between two identical textile antennas in an anechoic chamber.

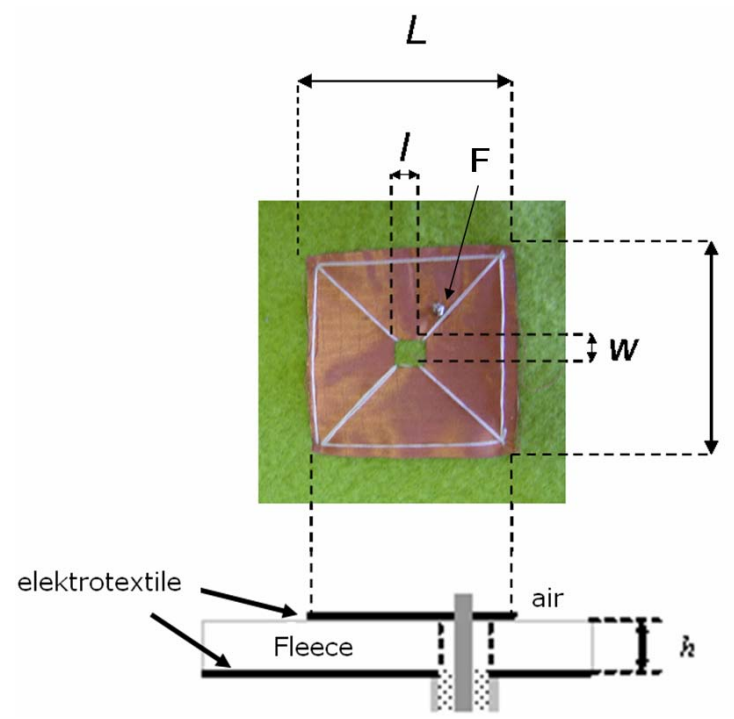

W

Figure 1. Geometry of the rectangular-ring textile antenna

\begin{tabular}{|c|ccccc|}
\hline Parameters & L & W & l & w & Feed point $F$ \\
\hline Value $[\mathrm{mm}]$ & 49 & 52 & 8 & 7 & $(7,9)$ \\
\hline
\end{tabular}

Table 1. Dimensions of the rectangular-ring antenna

\subsection{Construction of prototypes}

The rectangular-ring antenna of which the design parameters are shown in Fig. 1, has been simulated and optimised by means of the 2.5-D field simulator Advanced Design System (ADS)-Momentum from Agilent Technologies. The optimised parameters are shown in Table 1. The simulated bandwidth $(164 \mathrm{MHz})$ - this is the frequency band where $\left|S_{11}\right|<-10 \mathrm{~dB}$ - was designed to be larger than required for full coverage of the ISMband $(83.5 \mathrm{MHz})$, in order to accommodate changes in resonance frequency due to inaccuracies in the characterised substrate parameters and in the dimensions of the antenna. The first prototype, which relies on a glue stick to attach the different layers to one another, has been additionally stitched in order to obtain a uniform thickness. The second prototype uses an adhesive sheet which melts when ironing the layers that have to be attached. The results of the two prototypes are shown in Fig. 2. Both prototypes offer a larger bandwidth (first prototype: 228 $\mathrm{MHz}$, second prototype: $195 \mathrm{MHz}$ ) than predicted by simulation (164 MHz), indicating the presence of losses.

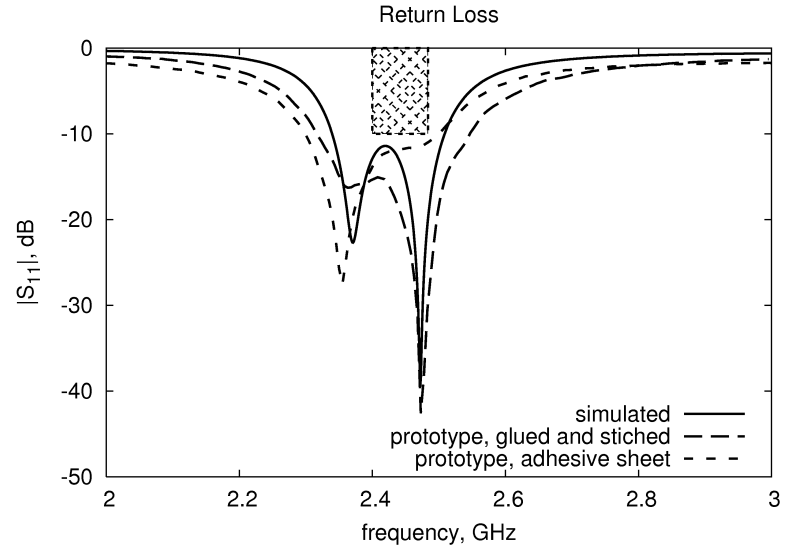

Figure 2. Simulated and measured return loss $\left(S_{11}\right)$

\subsection{Influence of finite ground plane and body}

During the design process based on field simulations using ADS-Momentum, a 2.5-D planar antenna with infinite ground plane and substrate was assumed. The influence of a finite ground plane with dimensions $(78 \mathrm{~mm} \times$ $73.5 \mathrm{~mm}$ ) on the antenna gain was simulated by means of the 3-D field simulator CST Microwave Studio. We also studied the effect of the human body, represented by a substrate with $\epsilon_{r}=42$ and $\sigma=0.99 \mathrm{~S} / \mathrm{m}$ located $8 \mathrm{~mm}$ below the antenna ground plane. The results for the antenna with finite ground plane located in free space (simulated in CST Microwave Studio as a 3-D antenna in air) and for the antenna with finite ground plane near the body (3-D antenna on body), together with the ADS-simulated gain assuming a 2.5-D planar antenna on an infinite ground plane, are shown in Fig. 3. Both the finite ground plane and the presence of the human body only have a marginal influence on the antenna gain.

\section{ANTENNA MEASUREMENTS}

\subsection{Directivity and Polarization}

Fig. 5 shows the directivity of a textile rectangular-ring antenna, assembled using an adhesive sheet, measured in a full-anechoic chamber at a frequency of $2.45 \mathrm{GHz}$. The ground plane dimensions of the prototype were chosen to be $11 \mathrm{~cm} \times 10 \mathrm{~cm}$. As shown in Fig. 5(a), in the XZplane (as defined in Fig. 4) the main beam is primarily oriented along the $z$-axis. At $2.45 \mathrm{GHz}$, a dominant linear polarization along the $y$-direction is seen. Yet, the polarization along the $x$-direction, which is about $6 \mathrm{~dB}$ lower at $2.45 \mathrm{GHz}$, remains sufficiently high to play a role when the transmitting and receiving textile antennas are tilted with respect the one another. This is proven in Section 3.2. The back radiation (i.e. the radiation for $\left.|\theta|>90^{\circ}\right)$ is limited and remains more than $20 \mathrm{~dB}$ below the main beam level. The same observations hold for the 


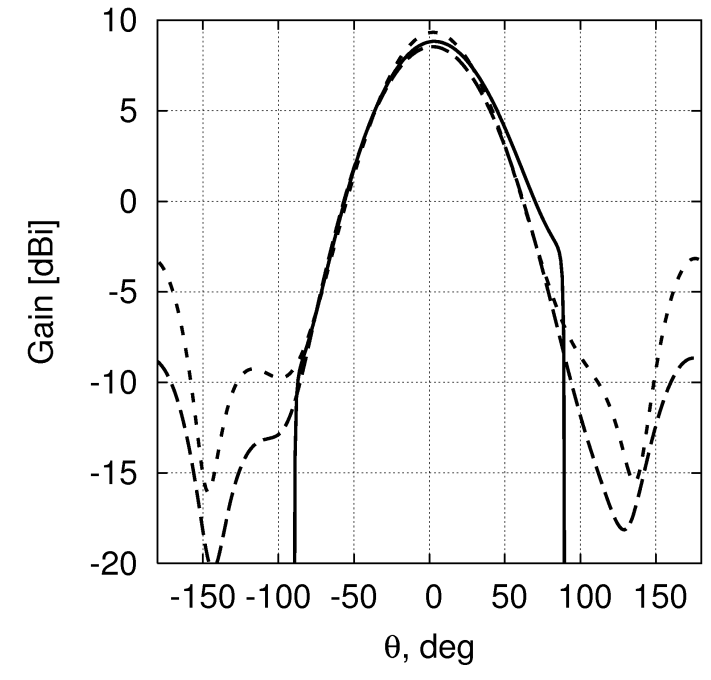

2.5-D planar antenna

3-D antenna in air - - -

3-D antenna on body - - - -

(a) XZ-plane, $2.45 \mathrm{GHz}$

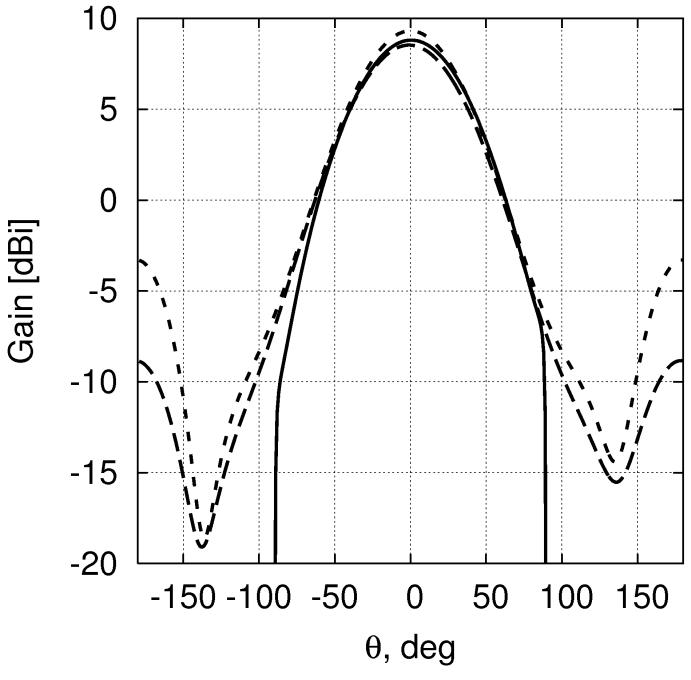

\section{5-D planar antenna 3-D antenna in air - - - - 3-D antenna on body - - - -}

(b) YZ-plane, $2.45 \mathrm{GHz}$

Figure 3. Influence of the finite ground plane and of the body on the gain

directivity measured in the $Y Z$-plane. However, one notices in Fig. 5(b) that the main beam direction has shifted to $\theta=14^{\circ}$.

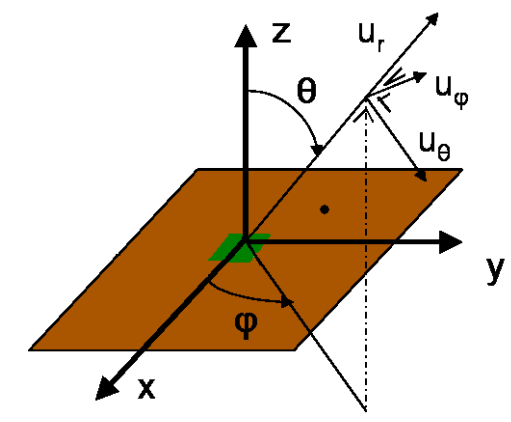

Figure 4. Antenna orientation for far-field measurements

\subsection{Antenna Gain and Efficiency}

The antenna gain is obtained by measuring the transmission between two antennas in an anechoic chamber. The first measurement yields the received power when both antennas have the same orientation, whereas the second measurement determines the received power when both antennas are rotated by 90 degrees with respect to each other. The measured gain of both prototypes for the two polarisations under consideration, together with the simulated gain, are shown in Fig. 6. The antenne gain of the first prototype is $1 \mathrm{~dB}$ higher than the gain of the second prototype, indicating that the adhesive sheet introduces extra substrate losses. Given that the antenna gain of the first prototype is less than $1.5 \mathrm{~dB}$ lower than the simulated gain, we obtain an antenna efficiency larger than $70 \%$ (compared to the $100 \%$ efficiency of the simulated lossless antenna). The antenna efficiency of the second prototype is $14 \%$ less than the first one, but with $56 \%$ still sufficient. For both prototypes, the measured gain is nearly the same in both configurations, indicating that the antennas are nearly circularly polarised.

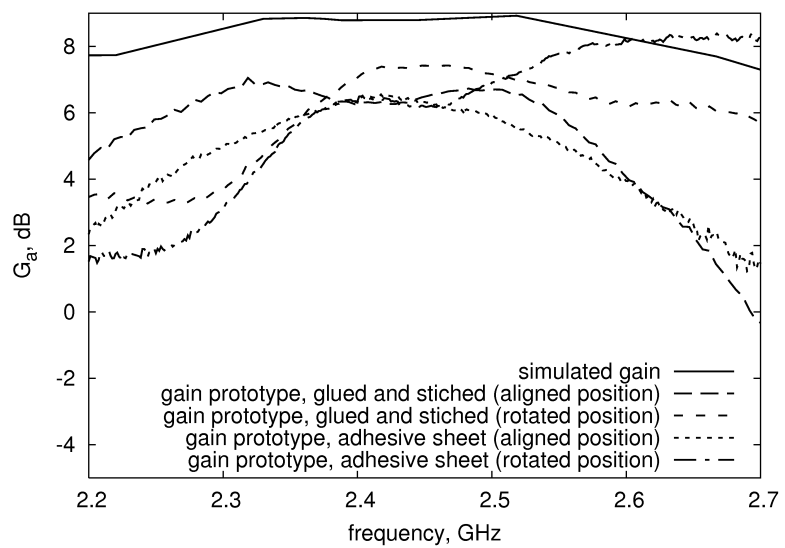

Figure 6. Simulated and measured gain of the prototypes 
(a) XZ-plane, $2.45 \mathrm{GHz}$

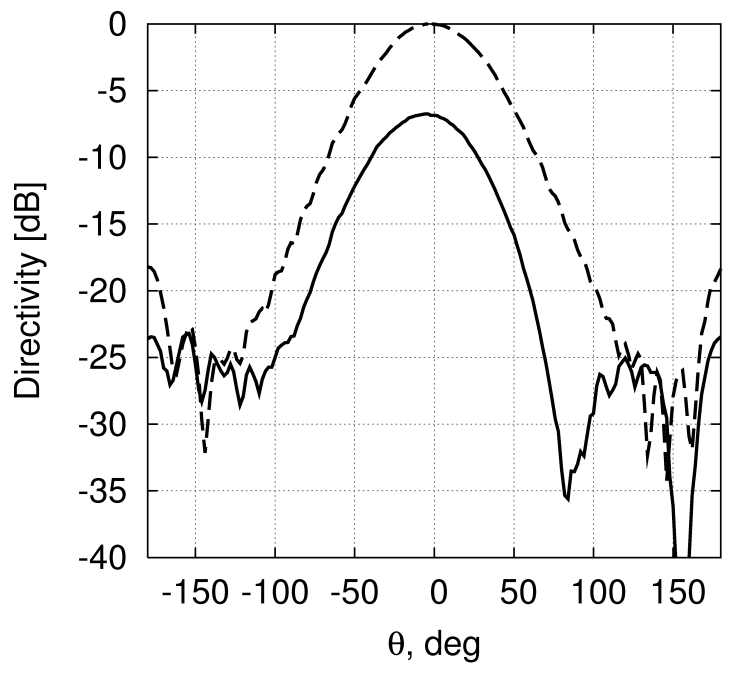

$\mathrm{E}_{\theta}-$

(a) XZ-plane, $2.45 \mathrm{GHz}$ (a) YZ-plane, $2.45 \mathrm{GHz}$

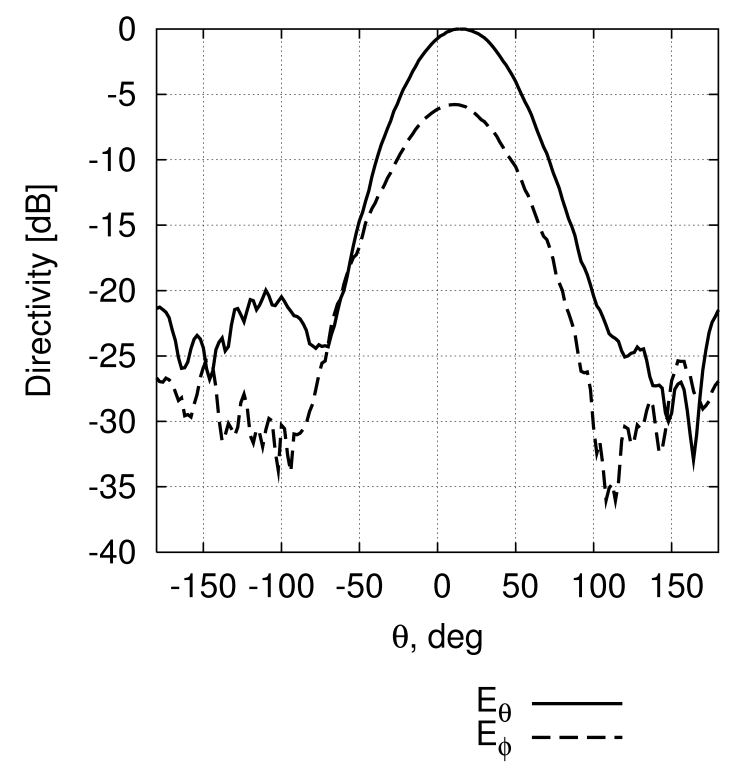

(b) YZ-plane, $2.45 \mathrm{GHz}$

Figure 5. Measured directivity

\subsection{Influence of bending}

In realistic applications the antenna is integrated into parts of clothes that can be bent. Therefore we attached the two prototypes to plastic cylinders with two diameters: $\mathrm{D}=12 \mathrm{~cm}$ and $\mathrm{D}=7.5 \mathrm{~cm}$. The results are shown in Fig. 7. In all cases bending causes a decrease in bandwidth, but because of the large bandwidth of the antenna, the ISM-band remains covered. Bending the first prototype results in the partial suppression of one mode, while this effect does not occur with the second prototype. This is the result of a better attachment of the layers because of the adhesive sheet.

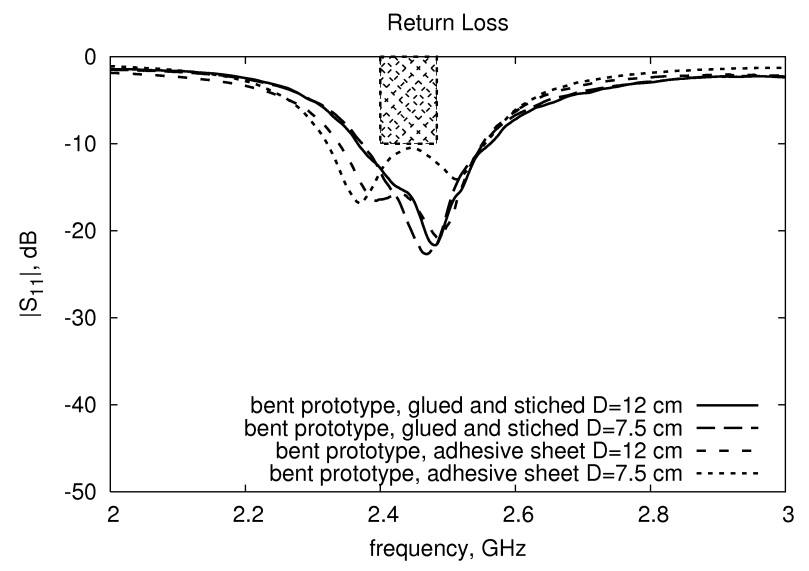

Figure 7. Influence of bending on the return loss $\left(S_{11}\right)$ of the prototypes

\subsection{Stability of the antenna characteristics in time}

After assembly, the glue used in the different prototypes will require some time to cure and the antenna characteristics can change during that process. Moreover, humidity can cause water to be trapped in the antenna substrate, which may lead to a shift in resonance frequency or a change of the antenna bandwidth. Finally, the conductivity of the electrotextiles and/or the connector could decrease because of oxidation. Therefore, in order to prove the stability of the antenna characteristics in time, we have meausured the return loss at different time instants. The result for the prototype assembled by glueing and stitching is shown in Fig. 8. Measurement 1 was performed a few days after construction, measurement 2 a few weeks after construction, whereas measurement 3 was performed six months after construction. Although some variation in the measured return loss is seen, especially in the antenna bandwidth, the antenna provides complete coverage of the ISM band over the complete time period under study. Similar conclusions can be drawn from Fig. 9, where the evolution of the return loss in time time is for the prototype assembled using the adhesive sheet. Again, measurement 1 was performed a few days after construction, measurement 2 one month after construction, whereas measurement 3 was performed two months after construction. A slight shift in resonance frequency is noticeable between the different measurements, as well as some variation in the antenna bandwidth. Yet, the design specification remain fulfilled during the complete time period under study. 


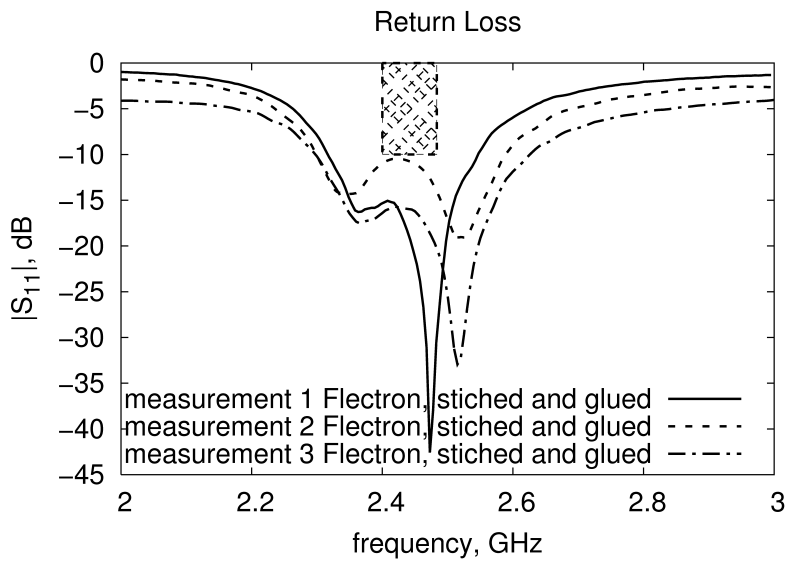

Figure 8. Return loss $\left(S_{11}\right)$ of the prototype assembled by glueing and stitching, measured at different time instants

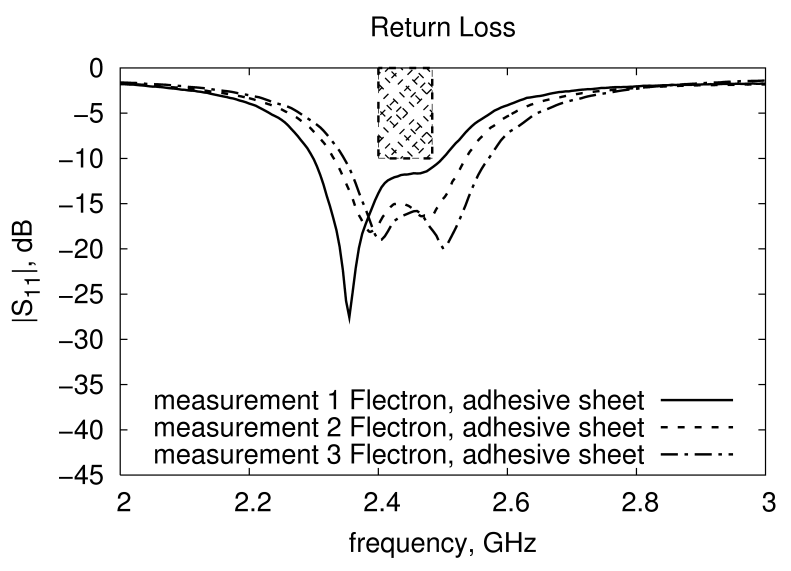

Figure 9. Return loss $\left(S_{11}\right)$ of the prototype with adhesive sheet, measured at different time instants

\section{CONCLUSIONS}

Two prototypes of a textile rectangular-ring antenna operating in the ISM-band were proposed. The glueing technique is preferred in wireless links requiring high antenna gain. When high-performance transceivers and receivers are used, the antennas assembled by means of an adhesive sheet are the preferred choice because of the better contact between the different layers. Obviously, the use of an adhesive sheet is also more suited to be applied in mass production. The antennas provide sufficient antenna gain for use in practical wireless links. They allow direct integration into garments.

\section{ACKNOWLEDGMENTS}

H. Rogier is a Postdoctoral Researcher of the FWOV. His research was supported by a grant of the
DWTC/SSTC, MOTION project.

\section{REFERENCES}

[1] P. Leitch and T. H. Tassarini. Interactive textiles: New materials in the new millenium, part 1. Journal of Industrial Textiles, 29(3):173-189, Mar. 2000.

[2] L. Van Langenhove, C. Hertleer, M. Catrysse, R. Puers, H. Van Egmond, and D. Matthys. Wearable eHealth Systems for Personalised Health Management, volume 108 of Studies in Health Technology and Informatics, chapter Smart Textiles, pages 344-352. IOS Press, 2004.

[3] M. Catrysse, R. Puers, C. Hertleer, L. Van Langenhove, H. Van Egmond, and D. Matthys. Towards the integration of textile sensors in a wireless monitoring suit. Sensors and Actuators, A-Physical, 114:302-311, 2004.

[4] L. Van Langenhove, R. Puers, and D. Matthys. Textiles for protection, chapter Intelligent textiles for protection, pages 176-193. Woodhead Publishing Limited, 2005.

[5] M. Klemm, I. Locher, and G. Tröster. A novel circularly polarized textile antenna for wearable applications. In Proc. of 7th European Microwave Week, pages 137-140, Amsterdam, Nederland, Oct 2004.

[6] P. Salonen, Y. Rahmat-Samii, H. Hurne, and M. Kivikoski. Dual-band wearable textile antenna. In Proc. of IEEE Antennas and Propagation Symposium, volume 1, pages 463-466, Monterey, USA, Jun 2004.

[7] P. Salonen, Y. Rahmat-Samii, and M. Kivikoski. Wearable antennas in the vicinity of human body. In Proc. of IEEE Antennas and Propagation Symposium, volume 1, pages 467-470, Monterey, USA, Jun 2004.

[8] C. Cibin, P. Leuchtmann, M. Gimersky, R. Vahldieck, and S. Moscibroda. A flexible wearable antenna. In Proc. of IEEE Antennas and Propagation Symposium, volume 4, pages 3589-3592, Monterey, USA, Jun 2004.

[9] A. Tronquo, H. Rogier, C. Hertleer, and L. Van Langenhove. A robust planar textile antenna for wireless body LANs operating in the $2.45-\mathrm{GHz}$ ISM band. IEE Electronics Letters, 42(3):142-143, Feb. 2006.

[10] C. Hertleer, F. De Clercq, A. Tronquo, H. Rogier, and L. Van Langenhove. Design of texile antennas for smart clothing. 2006 AUTEX Conference (Proceedings available on CDROM), pages 1-7, Jun 2006. 


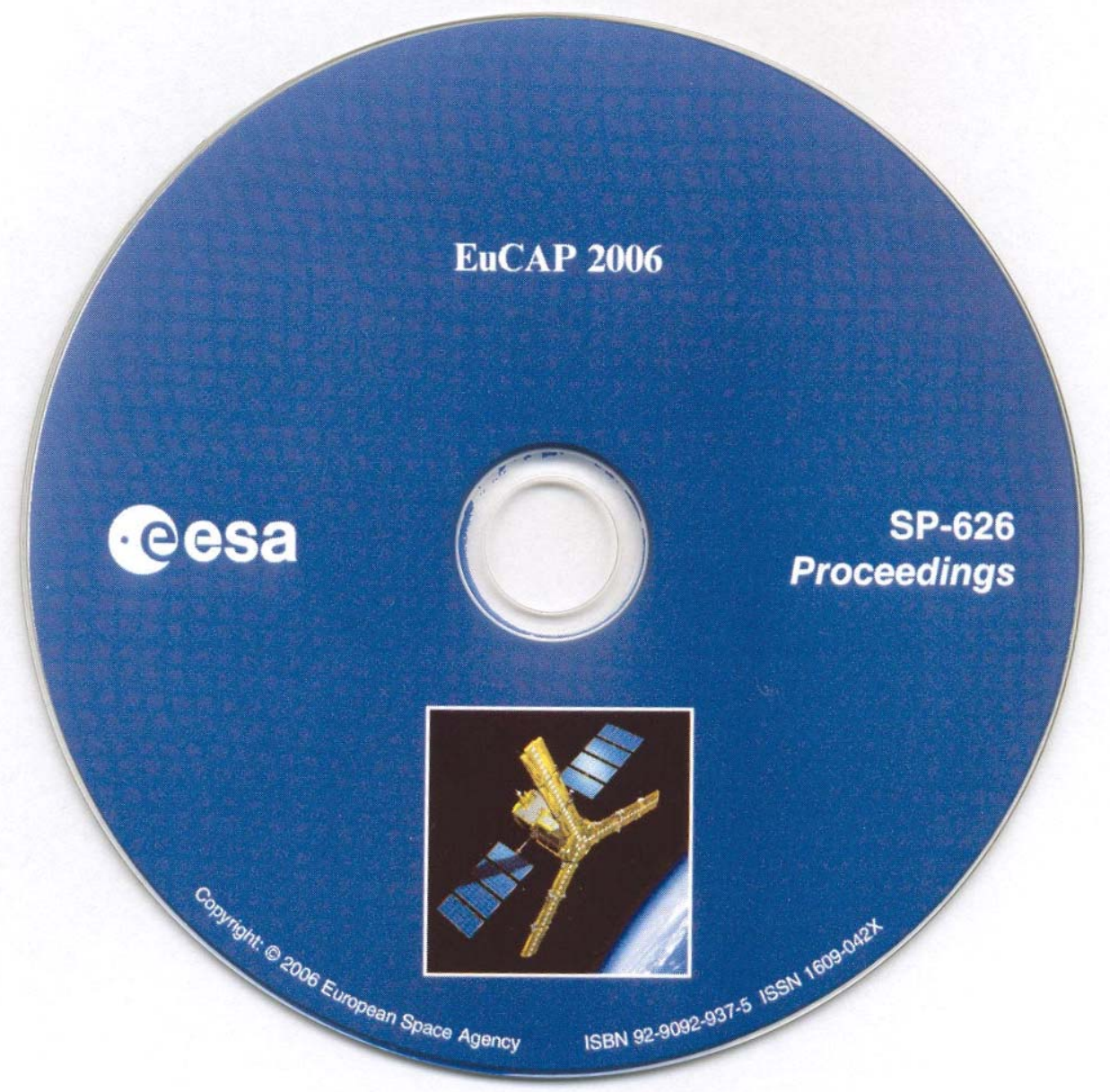




\section{EUCAP CHAIRS}

J. Mosig (General Chair), EPFL, CH

P. Ingvarson (Vice Chair), Saab Ericsson Space, $S$

B. Arbesse r-Rastburg (Vice Chair), ESA-ESTEC, NL

A. Roederer (Honorary Chair), ESA-ESTEC, NL

P. Brachat (Local Chair), France Telecom R\&D, F

C. Pichot (Local Chair), Univ. de Nice, F

Publication

Editors

Published by:

Price:

ISBN No:

ISSN No:

Copyright:
Proceedings of the European Conference on Antennas and Propagation 'EuCap 2006', Nice, France (ESA SP-626, October 2006)

H. Lacoste \& L. Ouwehand

ESA Publications Division

ESTEC

Postbus 299

2200 AG Noordwijk

The Netherlands

The Netherlands

60 Euros

92-9092-9375

1609-042X

(c) 2006 European Space Agency 
SP-626

October 2006

\author{
Proceedings of
}

\title{
The European Conference on Antennas and Propagation: EuCAP 2006
}

\author{
6-10 November 2006 \\ Nice, France
}

European Space Agency Agence spatiale européenne 